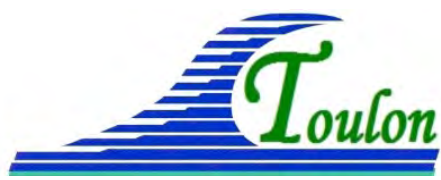

XIV èmes Journées Nationales Génie Côtier - Génie Civil Toulon, 29 juin au $1^{\text {er }}$ juillet 2016

DOI:10.5150/jngcgc.2016.062 (C) Editions Paralia CFL

disponible en ligne - http://www.paralia.fr - available online

\title{
Impact des activités de dragage et de clapage sur les communautés benthiques de substrat meuble de l'embouchure de l’Adour : résultats préliminaires
}

\section{Clémence FOULQUIER ${ }^{1}$, Didier RIHOUEY ${ }^{1}$, Floriane BOGUN ${ }^{1}$, Frank D'AMICO ${ }^{2}$, Thomas DE RECY ${ }^{3}$}

1. CASAGEC INGENIERIE, 18 rue Maryse Bastié, Zone de Maignon, 64600 Anglet, France.

foulquier@casagec.fr

2. UFR Sciences \& Techniques Côte Basque, Campus Montaury,

UMR CNRS n ${ }^{\circ} 5142$ Laboratoire de Mathématiques et de leurs Applications, FED 4155 MIRA "Milieux \& Ressources Aquatiques", F-64600 Anglet, France.

Frank.Damico@univ-pau.fr

3. CCI Bayonne Pays Basque, 50/51 allées Marines,

BP 215 - 64102 Bayonne cedex, France.

t.derecy@bayonne.cci.fr

\section{Résumé :}

Depuis les années 1980, les dragages du Port de Bayonne étaient organisés par un prestataire extérieur (marché public) en deux campagnes annuelles. En septembre 2015, la Chambre du Commerce et de l'Industrie de Bayonne Pays Basque (CCI BPB), gestionnaire du port de Bayonne et en charge des opérations de dragage, a acquis la drague mixte Hondarra pour l'entretien annuel des profondeurs. L'acquisition d'un navire à poste, en permettant une intervention toute l'année, risque de modifier l'impact des activités de dragage sur les habitats benthiques de substrat meuble. Les investigations biosédimentaires mises en œuvre depuis août 2014, soit un an avant l'arrivée de la nouvelle drague, ne permettent pas pour l'heure de conclure à un impact des opérations de dragage et d’immersion. En revanche, les résultats préliminaires soulignent l'importance des apports du fleuve sur la dynamique et la répartition des habitats benthiques présents aux abords et sur la zone d'immersion au large.

Mots-clés : Port estuarien de Bayonne, Dragage, Clapage, Impacts environnementaux, Habitats benthiques, Substrat meuble.

\section{Introduction}

\subsection{Situation géographique et contexte de l'étude}

Le port de Bayonne (Pyrénées-Atlantiques) se situe sur les deux rives de l’Adour aval. Ce port connait ainsi les contraintes classiques d'infrastructures estuariennes avec un ensablement à l'embouchure et un envasement récurrent du chenal et des souilles des 


\section{Thème 6 - Gestion durable des zones littorales et estuariennes}

postes d'accostage. Depuis des décennies, environ $500000 \mathrm{~m}^{3}$ de sable et $500000 \mathrm{~m}^{3}$ de vase sont respectivement dragués à l'embouchure et dans l'estuaire pour maintenir les profondeurs d'accès. Depuis les années 1980, le port de Bayonne ne dispose plus de drague à demeure et les dragages ont alors été organisés par l'Etat puis par un prestataire extérieur (marché public) en deux campagnes annuelles. La propriété du port de Bayonne a été transférée de l'État au Conseil Régional d’Aquitaine le 1er août 2006. Bayonne a ainsi été le premier port français d'intérêt national à devenir propriété d'une collectivité territoriale. Depuis 2010, la CCI de Bayonne Pays Basque (CCI BPB), gestionnaire du port de Bayonne, est en charge des opérations de dragage de maintien des profondeurs. En septembre 2015, la CCI BPB a acquis la drague mixte Hondarra qui assure désormais l'entretien des profondeurs tout au long de l'année.

Afin d'appréhender l'impact des activités de dragage en mode actualisé (drague à poste), CASAGEC INGENIERIE a établi un protocole de suivi des habitats benthiques de substrat meuble sur une zone englobant les sites intra-estuariens de dragage et les sites de clapage au large et à la côte au droit du littoral angloy (voir figure 2). Au travers de ce travail d'investigation de trois ans lancé en août 2014, l'objectif est dans un premier temps de déterminer la répartition et la dynamique des habitats benthiques au regard des conditions d'agitation et des apports de l'Adour. Dans un second temps, les données acquises devront permettre d'évaluer la capacité de recolonisation du milieu suite à la modification des fréquences et de la durée des dragages avec l'arrivée de la nouvelle drague à poste. Enfin, à plus large échelle, cette étude permettra d'acquérir des données et des connaissances sur les habitats benthiques de substrat meuble encore trop peu appréhendés sur le littoral basque dans sa partie française.

\subsection{Contexte hydrodynamique}

L'Adour prend sa source à environ $2200 \mathrm{~m}$ d'altitude, au pied du pic d'Espade, et se jette dans le golfe de Gascogne à Bayonne après avoir parcouru $312 \mathrm{~km}$. Ce fleuve, dont le bassin versant constitue une zone de près de $17000 \mathrm{~km}^{2}$, représente le cinquième réseau hydrographique de France. Sur la période 1970 à 2014, le débit moyen global de l'Adour est de $311 \mathrm{~m}^{3} / \mathrm{s}^{1}$. La crue de fréquence décennale $\left(\mathrm{Q}=2714 \mathrm{~m}^{3} / \mathrm{s}\right)$ a été atteinte ou dépassée 2 fois $(1974,1996)$ et la crue cinquantennale $\left(\mathrm{Q}=3469 \mathrm{~m}^{3} / \mathrm{s}\right)$ a été dépassée une fois en 2014 (3896 m³/s le 25 janvier 2014) (OEA, 2013).

Sur le littoral sud-aquitain, la marée est de type mésotidale semi-diurne, de période 12h25'. Le long du littoral basque, l'amplitude moyenne de la marée est de 3,85 m en vive-eau et de $1,85 \mathrm{~m}$ en morte-eau. Les courants induits par la marée gouvernent l'hydrodynamique intra-estuarienne dont l'intensité est directement influencée par l'amplitude de la marée et la morphologie de l'estuaire (courants $>2 \mathrm{~m} / \mathrm{s}^{1}$ dans les parties les plus étroites de l'estuaire pour un coefficient de 100). Au niveau de la zone de clapage au large, les courants de marée sont faibles à l'exception des périodes de crues où l'Adour peut engendrer des courants importants. En zone côtière, les courants 


\section{XIV èmes Journées Nationales Génie Côtier - Génie Civil \\ Toulon, 29 juin au $1^{\text {er }}$ juillet 2016}

induits par la marée sont négligeables devant ceux induits par le déferlement des vagues.

La côte Basque est exposée aux plus fortes houles du littoral français métropolitain du fait de sa position au fond du golfe de Gascogne et de la faible largeur du plateau continental. Les houles y sont de secteur ouest-nord-ouest dominant. Leur période moyenne est de 10s et leur hauteur significative moyenne de 2m (AUGRIS et al., 2009). Quelles que soient les directions de houle, le dépôt de clapage situé au large (voir figure 2) engendre une concentration de houle au sud de l'embouchure. Ce phénomène, couplé à la zone de calme créée par la présence de la digue nord, engendre un courant d'expansion dirigé vers le nord (ABADIE et al., 2006). Sur le reste du littoral angloy, les patrons de courant de déferlement sont essentiellement liés à la direction de la houle incidente et à la morphologie des barres sédimentaires.

\subsection{Contexte morphosédimentaire}

D’un point de vue morphosédimentaire, l'estuaire est essentiellement constitué de sables vaseux. Les vases (fines $<63 \mu \mathrm{m}$ ) sont présentes essentiellement sur les rives et plus particulièrement sur la rive droite. Plusieurs zones d'affleurements rocheux s'observent de l'amont vers l'aval (voir figure 1). L'embouchure est quant à elle exclusivement constituée de sables $(63 \mu \mathrm{m}<$ sables fins à moyens $<500 \mu \mathrm{m})$.

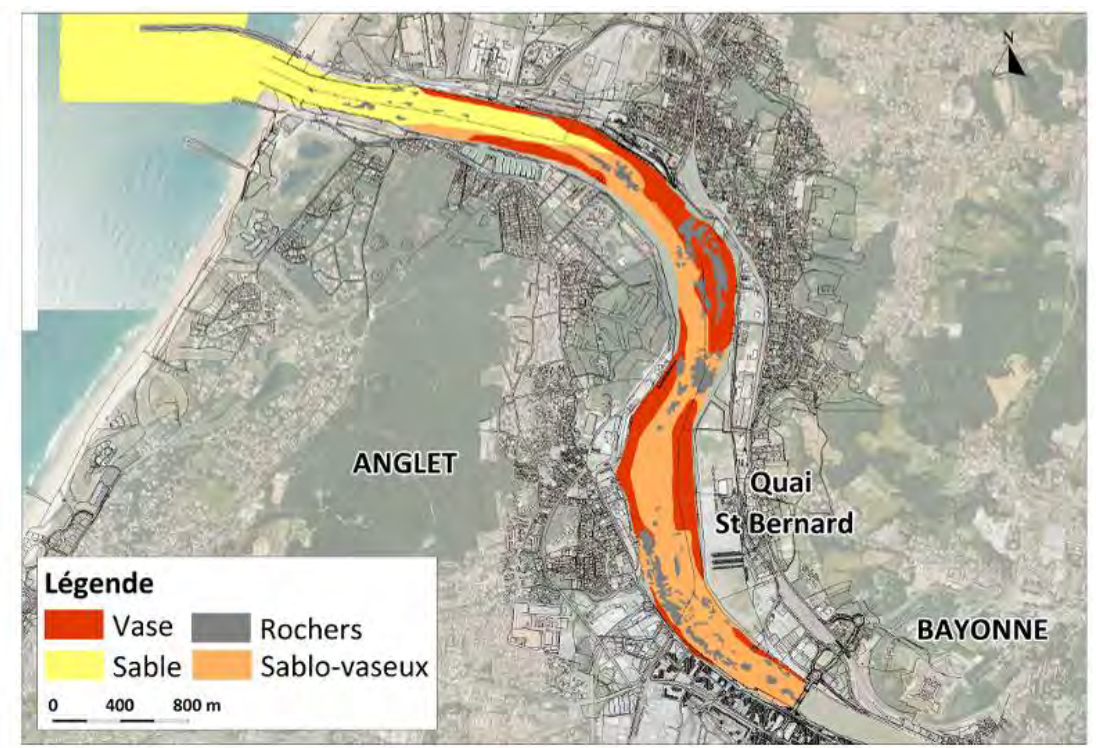

Figure 1. Faciès sédimentaires de l'Adour issus des prospections géophysiques (CASAGEC INGENIERIE, 2012).

En termes de dynamique sédimentaire intra-estuarienne, le flux sédimentaire de l'Adour (OEA, 2014) est conséquent (plus de 400000 t/an). La majorité de cet apport est réalisée en période hivernale et printanière, lors des évènements de crue. Les vitesses de 


\section{Thème 6 - Gestion durable des zones littorales et estuariennes}

sédimentations moyennes au niveau de la souille du quai St Bernard font clairement apparaitre une influence saisonnière avec des valeurs minimales et maximales se situant entre 3 et $20 \mathrm{~cm}$ par mois en été et 8 et $92 \mathrm{~cm}$ par mois en hiver (IMDC, 2010).

Dans la zone d'embouchure, il a été établi (ABADIE et al., 2006 ; RIHOUEY \& DUBRANNA, 2008) que seuls les évènements énergétiques, classifiés comme houles d'hiver et tempêtes ( $\mathrm{Hs}>4 \mathrm{~m}$ ), génèrent des circulations susceptibles de transporter des sédiments en provenance du sud. Ce processus d'ensablement engendre une forte évolution saisonnière des fonds avec des amplitudes faibles à modérées en été (entre $10000 \mathrm{~m}^{3}$ et $80000 \mathrm{~m}^{3}$ ) et des amplitudes modérées à fortes en hiver (entre $120000 \mathrm{~m}^{3}$ et $280000 \mathrm{~m}^{3}$ ) (CASAGEC INGENIERIE, 2014).

En zone littorale, le transit sédimentaire le long des plages d'Anglet ne présente pas de direction privilégiée. Les circulations fluctuent au gré des déplacements des barres sédimentaires (subtidale et intertidale). Les houles, de direction majoritaire frontale, engendrent de forts courants de retour (perpendiculaires à la côte) qui se chargent des matériaux les plus légers.

\subsection{Volumes de dragage et clapage sur la période 2014-2015}

Sur la période printemps 2014- printemps 2015, objet des premiers résultats préliminaires présentés ci-après (voir section 3), trois campagnes de dragage ont été mises en œuvre par le biais d’un prestataire extérieur (marché public) en deux campagnes annuelles (printemps et automne). Les volumes dragués puis clapés au cours de ces campagnes sont présentés dans le tableau ci-dessous :

Tableau 1. Volumes en $\mathrm{m}^{3}$ dragués puis clapés sur la période printemps 2014printemps 2015

\begin{tabular}{lllllll} 
& \multicolumn{3}{l}{ Volumes extraits totaux } & \multicolumn{4}{l}{ Volumes clapés totaux } \\
\cline { 2 - 7 } & Embouchure & $\begin{array}{l}\text { Saint } \\
\text { Bernard }\end{array}$ & Autres zones & $\begin{array}{l}\text { Zone } \\
\text { interne }\end{array}$ & $\begin{array}{l}\text { Zone du } \\
\text { large }\end{array}$ & $\begin{array}{l}\text { Zone } \\
\text { côtière }\end{array}$ \\
\hline $\begin{array}{l}\text { Printemps } \\
2014\end{array}$ & 406873 & 269142 & $\begin{array}{l}2983 \\
\text { (Zone 7) }\end{array}$ & 63080 & 478913 & 137005 \\
\hline $\begin{array}{l}\text { Automne } \\
2014\end{array}$ & 139332 & 199604 & $\begin{array}{l}8589 \\
\text { (Zone 7) }\end{array}$ & 6822 & 204175 & 136528 \\
\hline $\begin{array}{l}\text { Printemps } \\
2015\end{array}$ & 250655 & 203777 & $\begin{array}{l}\text { 5 085 } \\
\text { (Zones 6 et 7) }\end{array}$ & 26247 & 192442 & 240828 \\
\hline
\end{tabular}

L'essentiel des volumes dragués se concentre sur les zones de l'embouchure et de Saint Bernard (souille + zone d'évitage) représentant en moyenne plus de 98\% des quantités extraites. 


\section{XIV èmes Journées Nationales Génie Côtier - Génie Civil \\ Toulon, 29 juin au $1^{\text {er }}$ juillet 2016}

Les matériaux dragués sont ensuite immergés à l'arrêt sur les différentes zones de clapage. L'utilisation de la zone interne reste exceptionnelle et se limite aux conditions de mer ne permettant pas la sortie de la drague vers les zones de clapage du large et de la côte.

\section{Matériel et méthodes}

\subsection{Investigations biosédimentaires}

Au cours des 3 années de suivi (2014-2016), quatre campagnes de prélèvements (1 par saison) sont organisées par an au niveau de 20 stations d'échantillonnage (voir figure 2). Les prélèvements sont effectués à l'aide d'une benne Van Veen de surface $0,1 \mathrm{~m}^{2}$.

Trois zones de prospections se distinguent :

- la zone intra-estuarienne, lieu des opérations de dragage ;

- la zone côtière, particulièrement exposée aux houles et aux courants, où une partie des sables dragués à l'embouchure sont clapés ;

- la zone du large, site d'immersion des vases.

Au niveau de chaque point, 3 prélèvements sont voués aux analyses biologiques et à la mesure du potentiel d'oxydo-réduction. Un échantillon moyen de sédiment est également constitué pour l'analyse de la granulométrie et de la teneur en matière organique.

Les échantillons voués aux analyses biologiques sont tamisés à bord (maille carré de $1 \mathrm{~mm}$ ). Le refus de tamis est fixé à l'aide d'une solution d'éthanol. Au laboratoire, les différents invertébrés échantillonnés sont triés, comptabilisés et identifiés jusqu’à l'espèce sous loupe binoculaire et microscope. Les descripteurs écologiques utilisés ciaprès pour caractériser les communautés sont l’abondance et la richesse spécifique.

\subsection{Investigations géophysiques}

Des campagnes d'investigations géophysiques sont également mises en œuvre pour caractériser les paramètres physiques des différents habitats benthiques :

- un levé au sonar latéral à balayage et au sondeur à sédiment pour caractériser les différents faciès en présence ;

- des suivis bathymétriques pour appréhender la dynamique morphosédimentaire. 


\section{Thème 6 - Gestion durable des zones littorales et estuariennes}

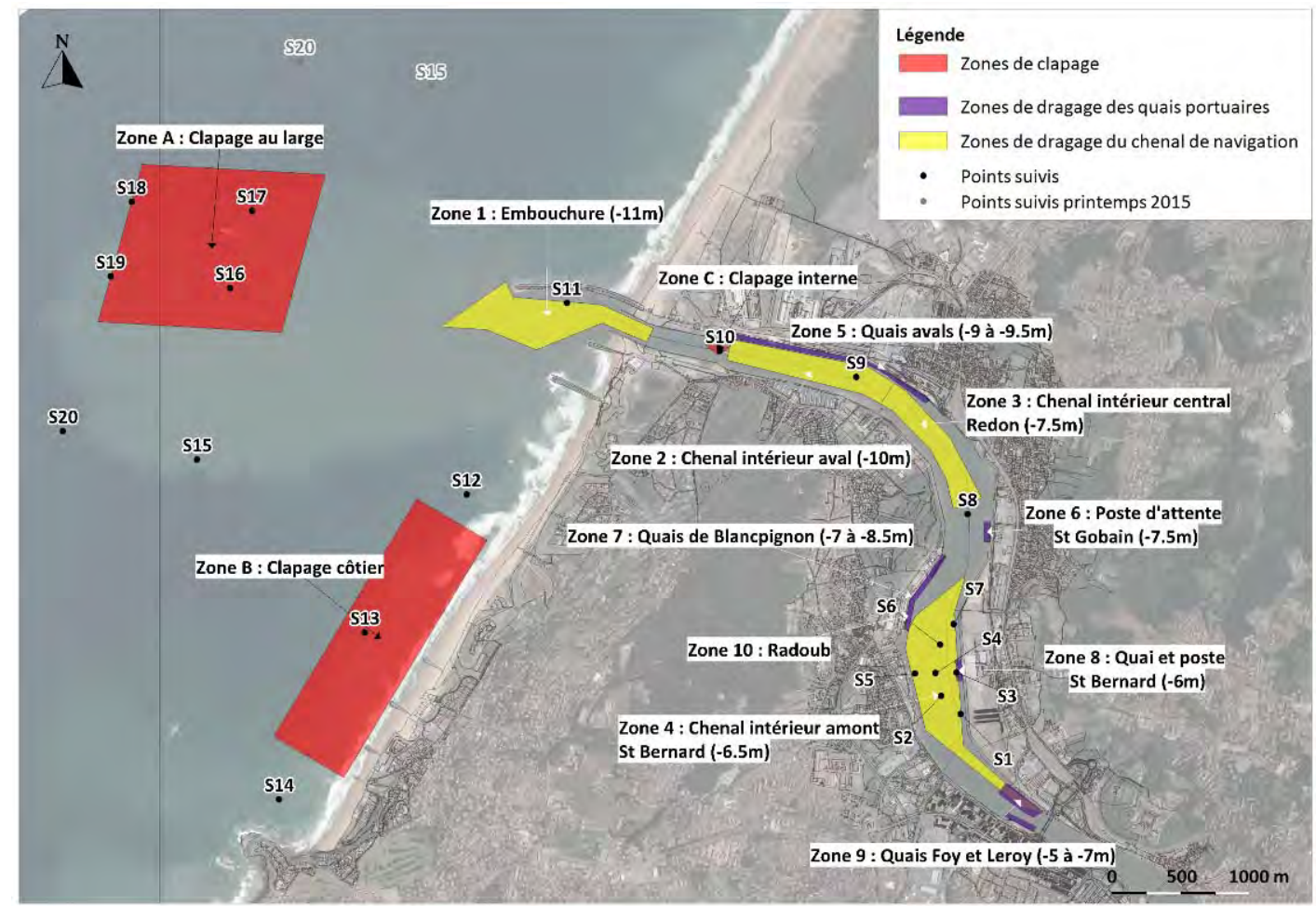

Figure 2. Localisation des zones de dragage et d'immersion et des points de prélèvements biosédimentaires. Les côtes minimales d'exploitation, exprimées en cote marine, sont renseignées entre parenthèses).

\section{Résultats préliminaires}

Les résultats présentés ci-après sont issus des 4 premières campagnes d'échantillonnage biosédimentaire mises en œuvre à l'été et l'automne 2014, à l'hiver 2014/2015 et au printemps 2015, soit respectivement 4 mois, 1 mois, 4 mois puis 1,5 mois après la dernière campagne de dragage. Les résultats des prospections géophysiques ne sont pas présentés dans cet article.

\subsection{Répartition des habitats benthiques de substrat meuble}

Les stations intra-estuariennes, localisées au niveau du banc St Bernard, zone générant l'essentiel des volumes de vases draguées $\left(400000 \mathrm{~m}^{3}\right)$, sont distribuées sur un faciès sablo-vaseux (40\% de fines en moyenne) à vaseux (80\% de fines en moyenne). Les taux de matière organique sont relativement forts avec une valeur moyenne de l'ordre de $5 \%$. Ces stations abritent une communauté C1 à Scrobicularia plana (voir figure 3) avec des abondances particulièrement importantes, atteignant les 2537 individus $/ \mathrm{m}^{2}$ à l'automne 2014 en station S5, et un nombre d'espèces restreint (voir tableau 2). Les autres espèces principales, présentes dans des proportions nettement inférieures, sont essentiellement euryhalines, telles que les polychètes Streblospio benedicti, Heteromastus filiformis et Hediste diversicolor ainsi que l'isopode Cyathura carinata. 


\section{XIV èmes Journées Nationales Génie Côtier - Génie Civil \\ Toulon, 29 juin au $1^{\text {er }}$ juillet 2016}

Tableau 2. Valeurs moyennes des paramètres physiques et biologiques permettant de décrire les 3 communautés observées au cours de la période août 2014 - juin 2015.

\begin{tabular}{|c|c|c|c|c|}
\hline & & \multicolumn{3}{|c|}{ Communautés macrobenthiques } \\
\hline & & C1 & C2 & C3 \\
\hline \multirow{2}{*}{$\begin{array}{l}\text { Paramètres } \\
\text { biologiques }\end{array}$} & Densité moyenne (ind. $/ 0,3 \mathrm{~m}^{2}$ ) & 217 & 22 & 34 \\
\hline & $\begin{array}{l}\text { Richesse spécifique moyenne (Nombre } \\
\text { d'espèces } / 0,3 \mathrm{~m}^{2} \text { ) }\end{array}$ & 5 & 6 & 11 \\
\hline \multirow{2}{*}{$\begin{array}{l}\text { Paramètres } \\
\text { physiques }\end{array}$} & $\%$ Fines $(\varnothing<63 \mu \mathrm{m})$ & 38 & 3 & 4 \\
\hline & Matière Organique (\%) & 4,9 & 0,8 & 1,0 \\
\hline
\end{tabular}

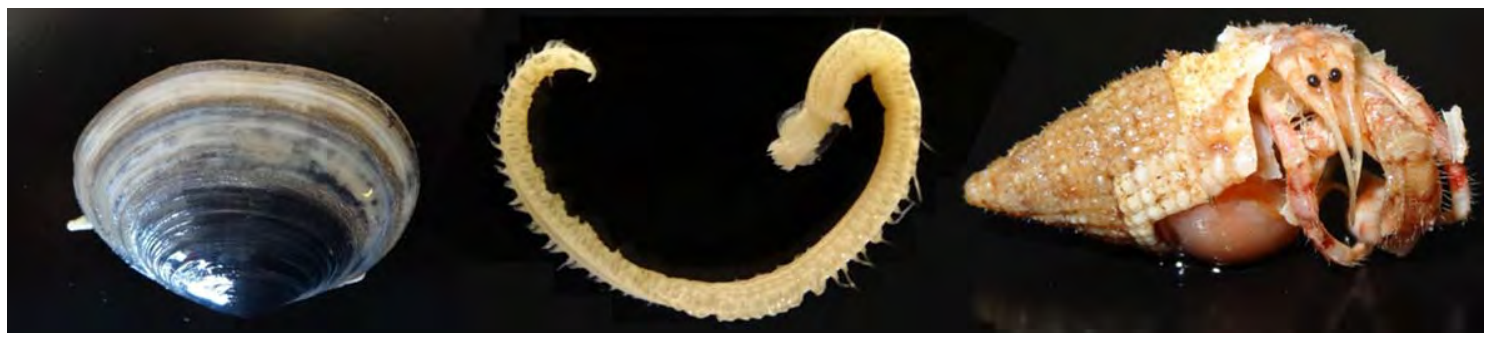

Figure 3. Espèces benthiques dominantes au sein des différentes communautés (de gauche à droite): Scrobicularia plana, Nephtys cirrosa, Diogenes pugilator.

Les stations côtières, situées au-droit des plages d'Anglet dans le périmètre d'action de la houle, se caractérisent par un faciès de sables fins avec une proportion non négligeable de sables moyens à grossiers (32\% en moyenne). Les taux de matière organique y sont particulièrement bas, de l'ordre de 0,75\%. Les invertébrés échantillonnés sur ces points constituent la communauté C2 à Scolelepis mesnilii et Nephtys cirrosa (voir figure 3). Elles se distinguent des autres zones prospectées par des valeurs d'abondances et de diversité particulièrement faibles en lien avec leur forte exposition aux houles.

Plus au large, les fonds sont composés en moyenne à 95\% de sables fins (60\%) à moyens (30\%) avec une proportion de fines de l'ordre de $5 \%$ hors évènement hydrologique majeur. La teneur moyenne en matière organique est de 1,2\%. Les stations échantillonnées sur cette zone abritent une communauté C3 dominée par le polychète Nephtys cirrosa, le pagure Diogenes pugilator (voir figure 3), l'échinoderme Echinocardium cordatum et le gastéropode Nassarius reticulatus.

3.2 Analyse préliminaire des facteurs influençant la dynamique et la répartition des habitats benthiques de substrat meuble

Les résultats préliminaires acquis au cours des 4 premières campagnes de prélèvements biosédimentaires ne permettent pas de conclure quant à un impact des activités de dragage et d'immersion. Dans le cadre de ces premières investigations l'influence des opérations n'apparait pas comme majeure. En effet, aucun échantillon biologique 


\section{Thème 6 - Gestion durable des zones littorales et estuariennes}

prélevé en zone de dragage et/ou de clapage n'était azoïque et ce quelque que soit la campagne de prélèvement et son écart temporel avec la période de travaux. De la même façon, aucune chute des paramètres abondance et richesse spécifique ou pic d'espèces opportunistes ne peut pour l'heure être relié aux actions de dragage et d'immersion.

En revanche, au regard de ces 4 premières campagnes, les premiers résultats traduisent la forte influence des apports de l'Adour sur la dynamique des habitats benthiques de la zone du large. Les fortes crues de 2014 ont engendré une profonde modification du substrat et de la macrofaune qu'il abrite. Une importante proportion de matériaux fins (> 60\% sur la station S20) a en effet été observée lors de la campagne d'août 2014 sur les stations du large. Ce substrat vaseux s'accompagne d'une explosion de l'abondance du bivalve Abra alba (8857 individus $/ \mathrm{m}^{2}$ en S16), espèce typique de la partie subtidale des estuaires vaseux du pays basque (BORJA et al., 2004) devenant dominante (contribution relative de 80\%) sur les stations du large en août 2014. Ce mollusque, caractéristique des faciès vaseux, est absent de l'ensemble des prélèvements effectués sur les stations du large lors des 3 campagnes suivantes (seuls deux individus ont été échantillonnés en décembre 2014). Par ailleurs, de telles proportions de vases n'ont jamais été observées lors des campagnes postérieures (voir figure 4). Lors des campagnes suivantes, seules des bioconstructions récifales (Sabellaria spinulosa) ont été échantillonnées sur les stations S15 et S20. Ces matériaux ne pouvant pas être assimilés à un substrat meuble, les deux stations ont été repositionnées lors de la $4{ }^{\text {ème }}$ campagne.

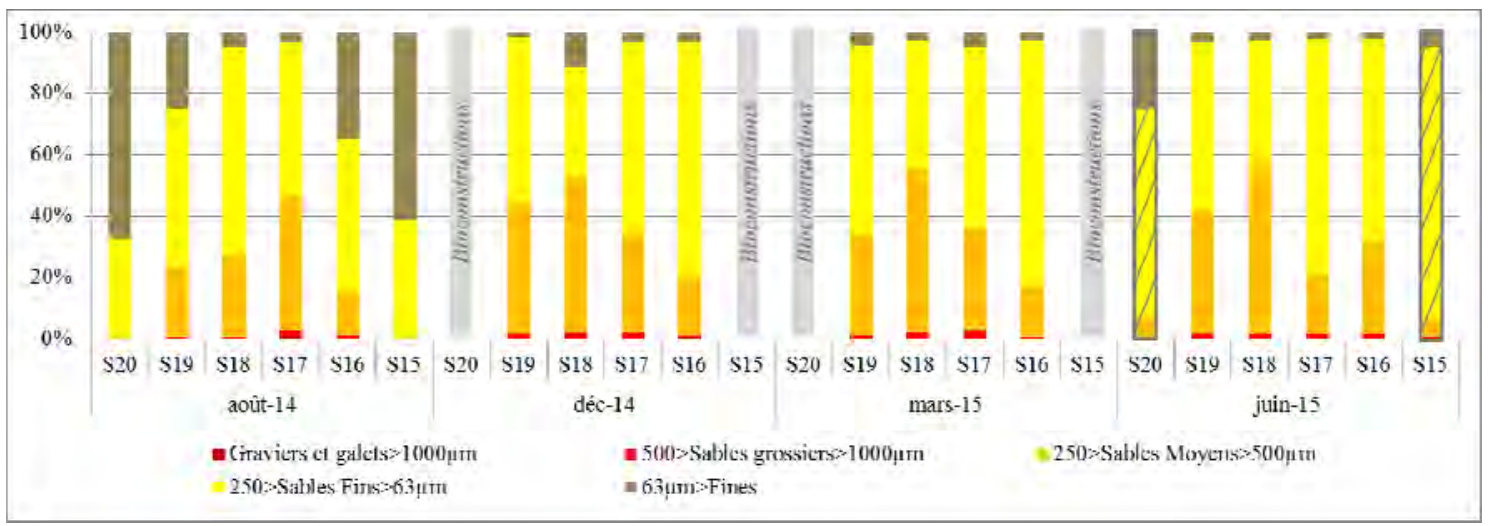

Figure 4. Evolution de la composition granulométrique des stations de la zone du large (les hachures indiquent une modification des coordonnées des stations S15 et S20).

\section{Conclusions}

Le suivi des habitats benthiques de substrat meuble au niveau du port de Bayonne et ses abords (zones de dragage et de clapage) a débuté en août 2014, soit un an avant l'arrivée de la drague à poste. Lors de cette période, les dragages étaient ainsi effectués via la mise œuvre de deux campagnes par an, au printemps et à l'automne. 


\section{XIV $V^{\text {èmes }}$ Journées Nationales Génie Côtier - Génie Civil \\ Toulon, 29 juin au $1^{\text {er }}$ juillet 2016}

Les 4 premières campagnes d'investigations biosédimentaires ont permis de conclure à une variabilité naturelle du milieu liée au régime hydrologique du fleuve. Les résultats soulignent en effet l'influence des apports de matières en provenance de l'Adour sur la répartition et la dynamique des habitats benthiques de substrat meuble de la zone du large. En revanche, ces résultats préliminaires ne permettent pas de conclure sur l'impact des activités de dragage et d'immersion du port de Bayonne. Pour l'heure, aucun lien ne peut en effet être établi entre les variations observées au sein des communautés macrobenthiques et les travaux de maintien des profondeurs du port.

La poursuite des campagnes biosédimentaires et l'exploitation croisée des données géophysiques permettront d'affiner la compréhension du milieu et ainsi de distinguer la variabilité naturelle de celle liée à la perturbation de l'écosystème. In fine, l'ensemble de ces éléments apportera un retour d'expérience sur l'impact de la modification des pratiques de dragage et améliorera l'état des connaissances des habitats benthiques de substrat meuble de l'estuaire de l'Adour.

\section{Références bibliographiques}

ABADIE S., BUTEL R., MAURIET S., MORICHON D., DUPUIS H. (2006). Wave climate and longshore drift on the South Aquitaine Coast, Continental Shelf Research, Vol. 26 (16), pp 1924-1939. http://dx.doi.org/10.1016/j.csr.2006.06.005

AUGRIS C., CAILL-MILLY N., DE CASAMAJOR M.N. (2009). Atlas thématique de l'environnement marin du pays basque et du Sud des Landes.

BORJA A., AGUIRREZABALAGA F., MARTÍNEZ J., SOLA J.C., GARCÍAARBERAS L., GOROSTIAGA J.M. (2004). Chapter 18 - Benthic communities, biogeography and resources management. Oceanography and Marine Environment of the Basque Country. Elsevier Oceanography Series (70), Ed. Borja A. et Collins M. , pp 531-547.

CASAGEC INGENIERIE (2014), Evolution interannuelle des zones de dragage. Note d'étude. Dossier de demande d'autorisation de dragage d'entretien et d'immersion du Port de Bayonne

IMDC (2010). Étude sur les moyens et méthodes d'entretien des profondeurs - Port de Bayonne. Phase 2 : Evaluation volumes et fréquences de dragage pour les scénarios d'étude.

OEA (2013). Note de travail de l'Observatoire de l'Estuaire de l'Adour: Caractérisation hydrodynamique de l'estuaire de l'Adour.

OEA (2014). Note Technique : Analyse du Flux Sédimentaire de l'Adour.

RIHOUEY D., DUBRANNA J. (2008). Modèle empirique 3D : application à la gestion des activités de dragage à l'embouchure de l'Adour. European Journal of Environmental and Civil Engineering, Vol. 12(1-2), pp.133-146. 
Thème 6 - Gestion durable des zones littorales et estuariennes 